\title{
Fundamental limits of laser power stabilization via a radiation pressure transfer scheme
}

\author{
Marina Trad Nery, ${ }^{1, *}$ Stefan L. Danilishin, ${ }^{2}$ Jasper R. Venneberg, ${ }^{1}$ and \\ BENNO WILLKE ${ }^{1}$ (1) \\ ${ }^{1}$ Max-Planck-Institut für Gravitationsphysik (Albert-Einstein-Institut) and Leibniz Universität Hannover, 30167 Hannover, Germany \\ ${ }^{2}$ GWFP, Faculty of Science and Engineering, Maastricht University, 6200 MD Maastricht, Netherlands \\ *Corresponding author: marina.trad@aei.mpg.de
}

Received 8 April 2020; revised 9 June 2020; accepted 13 June 2020; posted 15 June 2020 (Doc. ID 394547); published 10 July 2020

\begin{abstract}
Traditional active laser power stabilization schemes are fundamentally limited by quantum shot noise on the in-loop photodetector. One way to overcome this limitation is to implement a nondemolition sensing scheme where laser power fluctuations are transferred to motion of a microoscillator, which can be sensed with a high signal-to-noise ratio. In this Letter, we analyze the power stability achievable in a nondemolition scheme limited by quantum and thermal noise. Under the assumption of realistic experimental parameters, we show that generation of a strong bright squeezed quantum state of light should be possible. () 2020 Optical Society of America
\end{abstract}

https://doi.org/10.1364/OL.394547

Provided under the terms of the OSA Open Access Publishing Agreement Many modern high-precision metrology experiments require high laser power stability. Interferometric gravitational wave detectors [1], for example, have one of the most demanding requirements at low frequencies where a relative power noise of $2 \times 10^{-9} \mathrm{~Hz}^{-1 / 2}$ is needed at $10 \mathrm{~Hz}$. Such requirements are fulfilled with active stabilization schemes where a negative feedback control loop is implemented. The most common scheme is the traditional scheme, where a fraction of the laser light is sensed by a photodetector and compared to a stable reference. This scheme is fundamentally limited by shot noise of the detected light, which couples as sensor noise in the control loop and therefore is imprinted in the out-of-loop beam. The straightforward way to increase the signal-to-noise ratio in the in-loop detector is to increase the detected power $P$. However, since the signal-to-noise ratio scales with $P^{-1 / 2}$, a photocurrent of at least $200 \mathrm{~mA}$ needs to be detected to meet the stated requirements. This high photocurrent imposes significant technical challenges due to high thermal loads on the photodetector.

Alternative routes were adopted to circumvent these limitations such as substituting the single diode for an array of photodiodes [2], injecting squeezed light in the detected beam [3], and sensing power fluctuations in reflection of an optical cavity (OAC-coupling) [4]. Another approach is to implement a nondemolition scheme in which power fluctuations are transferred to another observable of the light field, which is then measured with low sensing noise. The first proposal and experimental realization of such a scheme was done in 1986 [5] with a nonlinear crystal as the transfer medium. In this Letter, we analyze a nondemolition scheme where power fluctuations of a strong transfer beam are transferred to motion of a microoscillator mirror. A similar scheme was analyzed in [6], but for a freely suspended mirror inside an optical cavity. Here the micro-oscillator is used as an end mirror of a Michelson interferometer whose output signal is fed-back to the laser power. The interferometer represents the in-loop sensor of this scheme, and therefore interferometer noise sources will be imprinted in the out-of-loop beam, setting a lower limit to the final power stability. We determine this limit for an interferometer fundamentally limited by quantum and thermal noise.

The details of the stabilization scheme are shown in Fig. 1. The free running field $\hat{f}$ to be stabilized from the transfer beam (red trace) is sent to an amplitude modulator, and the transmitted field $\hat{t}$ impinges on a micro-oscillator and is fully reflected to the out-of-loop field $\hat{o}$. The micro-oscillator's position $\hat{x}$ is sensed via a Michelson interferometer with a balanced homodyne readout [7] ideally sensitive only to the phase quadrature of the output field $\hat{b}$, which represents the differential length of the interferometer arms. This is a realistic approximation for a homodyne detector with high common mode rejection and good phase stability. The output signal is then amplified by a complex gain $\epsilon$, and the resulting feedback signal is sent to the amplitude modulator. The interferometer implements a weak sensing beam (orange trace) with a field $\hat{s}$, which is provided by an independent laser source. The figure also shows the vacuum field $\hat{v}$ that couples at the dark port of the interferometer.

The quantum limit of the proposed setup was calculated with a standard two-photon description [7-9] of the electric strain operator of a light wave using amplitude (c-subscript) and phase (s-subscript) quadrature amplitudes. In the frequency domain, the two-photon quadrature operators are written in terms of the creation $\hat{a}$ and annihilation $\hat{a}^{\dagger}$ operators for the upper and lower modulation sidebands as

$$
\hat{a}_{\mathrm{c}}(\Omega)=\frac{\hat{a}_{\omega_{0}+\Omega}+\hat{a}_{\omega_{0}-\Omega}^{\dagger}}{\sqrt{2}} \text { and } \quad \hat{a}_{\mathrm{s}}(\Omega)=\frac{\hat{a}_{\omega_{0}+\Omega}-\hat{a}_{\omega_{0}-\Omega}^{\dagger}}{i \sqrt{2}} \text {, }
$$




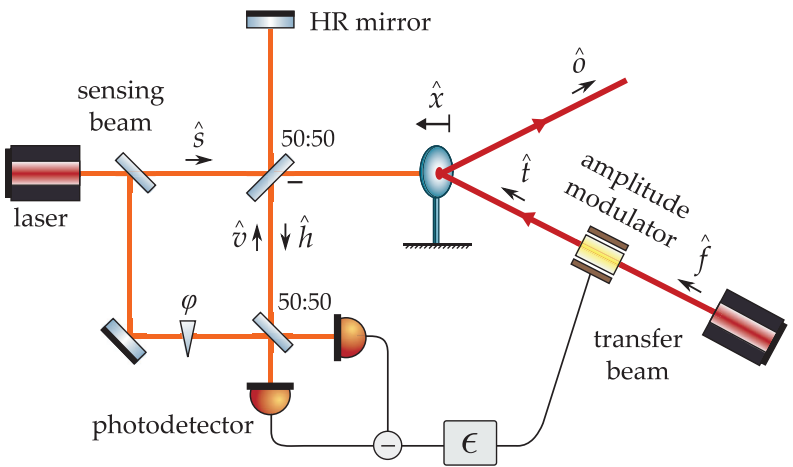

Fig. 1. Schematic of the stabilization scheme via radiation pressure. Power fluctuations of a strong transfer beam are transferred to motion of a micro-oscillator mirror. The micro-oscillator's position is sensed via a Michelson interferometer with a balanced homodyne readout and a weak sensing beam. The feedback signal is amplified by a gain $\epsilon$. The convention adopted as the positive displacement direction $\hat{x}$ is shown by the arrow on top of the oscillator.

To calculate the amplitude and phase quadratures of the out-of-loop beam, we should determine the relation between the input and output light quadratures and the oscillator displacement $\hat{x}$. The oscillator responds to the radiation pressure force like a harmonic oscillator with a mechanical susceptibility of

$$
\chi_{m}(\Omega)=\frac{1}{-m \Omega^{2}+m \Omega_{0}^{2}(1+i \phi)},
$$

where $m$ is the mass, $\Omega_{0}$ is the fundamental resonance frequency, and $\phi$ is the loss angle. The position is influenced by the two independent fields $\hat{s}$ and $\hat{v}$ in the interferometer, and by the stabilized field $\hat{t}$,

$$
\hat{x}=\chi_{m}\left(-\alpha \cdot\left(\hat{s}_{\mathrm{c}}-\hat{v}_{\mathrm{c}}\right)+\beta \cdot \hat{t}_{\mathrm{c}}\right),
$$

where $\alpha$ and $\beta$ are constants that depend on the mean power of the sensing $\left(\bar{P}_{\mathrm{s}}\right)$ and transfer $\left(\bar{P}_{\mathrm{t}}\right)$ beams,

$$
\alpha=\sqrt{2 \bar{P}_{\mathrm{s}} \hbar \omega_{0}} / c \text { and } \beta=\sqrt{8 \bar{P}_{\mathrm{t}} \hbar \omega_{0}} / c
$$

for a $100 \%$ reflective mirror. For the chosen readout scheme, the feedback signal is proportional to the phase quadrature of the output field $\hat{h}$ of the interferometer,

$$
\hat{h}_{\mathrm{s}}=-\hat{v}_{\mathrm{s}}-\alpha \cdot \hat{x} / \hbar=-\hat{v}_{\mathrm{s}}+K_{\alpha} \cdot\left(\hat{s}_{\mathrm{c}}-\hat{v}_{\mathrm{c}}\right)-\sqrt{K_{\alpha} K_{\beta}} \cdot \hat{t}_{\mathrm{c}}
$$

where $K_{\alpha}=\alpha^{2} \chi_{m} / \hbar$ and $K_{\beta}=\beta^{2} \chi_{m} / \hbar$ are the optomechanical coupling strengths [10], which express the transfer function from amplitude to phase quadrature driven by radiation pressure. The amplitude quadrature transmitted by the modulator is given by $\hat{t}_{\mathrm{c}}=\hat{f}_{\mathrm{c}}+\epsilon \cdot \hat{h}_{\mathrm{s}}$, which, in the steady state regime, can be combined with Eq. (5) to obtain

$$
\hat{t}_{\mathrm{c}}=\frac{\hat{f}_{\mathrm{c}}+\epsilon\left(-\hat{v}_{\mathrm{s}}+K_{\alpha} \cdot\left(\hat{s}_{\mathrm{c}}-\hat{v}_{\mathrm{c}}\right)\right)}{1+\epsilon \sqrt{K_{\alpha} K_{\beta}}} \quad \text { and } \quad \hat{t}_{\mathrm{s}}=\hat{f}_{\mathrm{s}}
$$

Here an ideal modulator acting only in the amplitude quadrature of the light was considered. The factor $\epsilon \sqrt{K_{\alpha} K_{\beta}}$ corresponds to the open loop gain of the feedback control loop.
Under the assumption that the mirror displacement is small such that $x \ll \lambda$, the out-of-loop quadratures can be written as

$$
\hat{o}_{\mathrm{c}}=\hat{t}_{\mathrm{c}} \quad \text { and } \quad \hat{o}_{\mathrm{s}}=\hat{t}_{\mathrm{s}}-\beta \cdot \hat{x} / \hbar
$$

which, according to Eq. (6), are given as functions of the uncorrelated input fields as

$$
\begin{gathered}
\hat{o}_{\mathrm{c}}=\frac{\hat{f}_{\mathrm{c}}-\epsilon \cdot \hat{v}_{\mathrm{s}}+\epsilon K_{\alpha} \cdot\left(\hat{s}_{\mathrm{c}}-\hat{v}_{\mathrm{c}}\right)}{1+\epsilon \sqrt{K_{\alpha} K_{\beta}}}, \\
\hat{o}_{\mathrm{s}}=\hat{f}_{\mathrm{s}}-\frac{K_{\beta} \cdot \hat{f}_{\mathrm{c}}-\epsilon K_{\beta} \cdot \hat{v}_{\mathrm{s}}-\sqrt{K_{\alpha} K_{\beta}} \cdot\left(\hat{s}_{\mathrm{c}}-\hat{v}_{\mathrm{c}}\right)}{1+\epsilon \sqrt{K_{\alpha} K_{\beta}}} .
\end{gathered}
$$

As expected, the free running noise $\hat{f}_{\mathrm{c}}$ is reduced arbitrarily by increasing the feedback gain $|\epsilon|$ and the transfer magnitude $\left|K_{\alpha} K_{\beta}\right|$. Additionally, the readout and radiation pressure noise introduced by the interferometer [second and third terms in Eq. (8)] are imprinted in the out-of-loop field and cannot be decreased below a certain value. The single-sided power spectral densities (PSDs) [7] of the amplitude $\left(S_{\mathrm{cc}}\right)$ and phase quadratures $\left(S_{\text {ss }}\right)$ of the out-of-loop beam are, respectively,

$$
\begin{gathered}
S_{\mathrm{cc}}^{\hat{o}}=\frac{S_{\mathrm{cc}}^{\hat{f}}+|\epsilon|^{2}+\left|\epsilon K_{\alpha}\right|^{2}\left(S_{\mathrm{cc}}^{\hat{s}}+1\right)}{\left|1+\epsilon \sqrt{K_{\alpha} K_{\beta}}\right|^{2}}, \\
S_{\mathrm{ss}}^{\hat{o}}=S_{\mathrm{ss}}^{\hat{f}}+\frac{\left|K_{\beta}\right|^{2}\left(S_{\mathrm{cc}}^{\hat{f}}+|\epsilon|^{2}\right)+\left|K_{\alpha} K_{\beta}\right|\left(S_{\mathrm{cc}}^{\hat{s}}+1\right)}{\left|1+\epsilon \sqrt{K_{\alpha} K_{\beta}}\right|^{2}} .
\end{gathered}
$$

To understand the scheme dynamics, let us assume that the sensing and transfer beams are initially in a coherent state, i.e., $S_{\mathrm{cc}}^{\hat{f}(\hat{s})}=1$ and $S_{\mathrm{ss}}^{\hat{f}(\hat{s})}=1$. Figure 2 shows the amplitude spectral density (ASD) of the amplitude (upper plot) and phase (lower plot) quadratures, calculated for coherent states, for different amplification factors $\epsilon$ (treated as frequency independent). The micro-oscillator chosen for all analysis in this Letter has a mass of $m=40 \mathrm{ng}$, fundamental resonance frequency $\Omega_{0}=2 \pi \times 100 \mathrm{~Hz}$, and a structural quality factor $Q=2 \times 10^{5}$. More details about this choice are given in later. Pitch and yaw modes of the oscillator should not couple in the measurement if the beams are centered to the oscillator's mirror pad. The wavelengths of the transfer and sensing beams were set to $1064 \mathrm{~nm}$.

First let us analyze the amplitude quadrature. When the loop is open $(\epsilon=0)$, the out-of-loop ASD is the same as the ASD of $\hat{f}_{\mathrm{c}}$, which is frequency independent and equal to 1 for a coherent state. When the loop is closed, an interesting result is obtained: for low frequencies, the amplitude quadrature is squeezed, as it has an ASD smaller than the vacuum state, whereas for high frequencies, extra noise above the vacuum level is always imprinted. This behavior can be better understood by setting the limit of $|\epsilon| \rightarrow \infty$ in Eq. (8). The free running amplitude quadrature $\hat{f}_{\mathrm{c}}$ is completely suppressed by the high loop gain and has no contribution to $\hat{o}_{\mathrm{c}}$. The radiation pressure noise contribution, caused by $\hat{v}_{\mathrm{c}}$ and $\hat{s}_{\mathrm{c}}$, is constant and depends only on the ratio between the sensing and transfer beam mean powers, which can be set to much smaller than 1 . The interferometer readout noise contribution, caused by $\hat{v}_{\mathrm{s}}$, is divided by the oscillator's susceptibility since $\sqrt{K_{\alpha} K_{\beta}}=\alpha \beta \chi_{m} / \hbar$. This means that below $\Omega_{0}$, the readout noise is suppressed by a constant and large factor, while above $\Omega_{0}$, the suppression gets smaller and is 

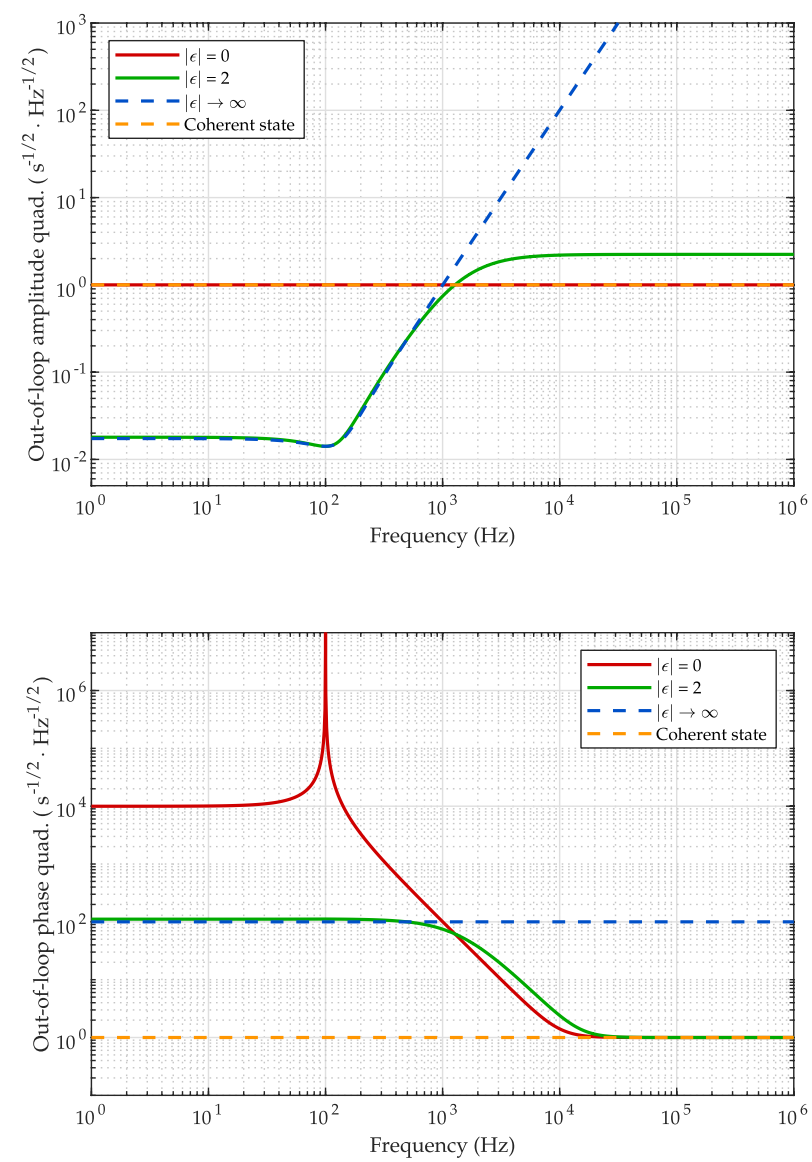

Fig. 2. ASD of $S_{\mathrm{cc}}^{\hat{o}}$ (upper plot) and $S_{\mathrm{ss}}^{\hat{o}}$ (lower plot) calculated for different amplification factors $\epsilon$ and for $\bar{P}_{\mathrm{t}}=1 \mathrm{~W}$ and $\bar{P}_{\mathrm{s}}=0.4 \mathrm{~mW}$. The ASD of a coherent state is displayed by the dashed yellow line as a reference.

subsequently amplified in a $\Omega^{2}$ fashion, following the decrease in the susceptibility proportional to $1 / \Omega^{2}$. This amplification happens because, since the micro-oscillator response at high frequencies gets smaller, the loop needs to apply a higher power modulation on the transfer beam to compensate the sensor noise and keep the error signal zero.

It is important to note that the generation of amplitude squeezing via the feedback loop in this scheme does not violate the Weisman and Milburn [11] condition, which states that "feedback mediated by homodyne detection can only produce nonclassical light, if the system dynamics can do so without feedback," since optomechanical systems are known to produce ponderomotive squeezing [12] on their own.

The Heisenberg uncertainty principle stipulates that at frequencies where the amplitude quadrature is squeezed, the phase quadrature must be antisqueezed. This is exactly what is shown in the lower plot of Fig. 2. When the loop is open, the phase quadrature has contributions from $\hat{f}_{\mathrm{s}}$, plus a frequencydependent term caused by the radiation pressure displacement due to $\hat{f}_{\mathrm{c}}, \hat{s}_{\mathrm{c}}$, and $\hat{v}_{\mathrm{c}}$, which is shaped by the oscillator's susceptibility. When the loop is closed, the amplitude quadrature $\hat{t}_{\mathrm{c}}$ is suppressed at low frequencies, which consequently leads to the reduction in the phase quadrature $\hat{o}_{s}$ as well. However, unlike the amplitude, the phase quadrature will never undergo squeezing since there is no mechanism in the setup to suppress $\hat{f}_{\mathrm{s}}$. In the regime where $|\epsilon| \rightarrow \infty$, an additional constant noise is imprinted on the phase quadrature at high frequencies. This is because the amplitude quadrature $\hat{t}_{\mathrm{c}}$ increases with $\Omega^{2}$ while the susceptibility decreases with $1 / \Omega^{2}$, making the result frequency independent.

The uncertainty principle is always satisfied, which can be easier seen in the regime where $|\epsilon| \rightarrow \infty$,

$$
S_{\mathrm{cc}}^{\hat{o}} S_{\mathrm{ss}}^{\hat{o}}-\left|S_{\mathrm{cs}}^{\hat{o}}\right|^{2}=\left|K_{\alpha} K_{\beta}\right|^{-1}+2\left|K_{\alpha} K_{\beta}^{-1}\right|+2>1 .
$$

The assumption made previously that the transfer and sensing beams are in a coherent state was useful to understand the scheme, but it is unrealistic since lasers always exhibit technical noise. Therefore, the power stability in the out-of-loop beam is analyzed taking into account the initial technical power noise in both beams. The PSD of the out-of-loop beam relative power noise $\left(\mathrm{RPN}_{\mathrm{ool}, \mathrm{q}}\right)$ as a function of the relative power noise in the sensing and transfer beams $\left(\mathrm{RPN}_{\mathrm{s}}\right.$ and $\left.\mathrm{RPN}_{\mathrm{f}}\right)$ can be calculated by combining Eq. (10) with

$$
\mathrm{RPN}_{j}=\frac{\delta P_{j}}{\bar{P}_{j}}=\sqrt{\frac{2 \hbar \omega_{0} S_{\mathrm{cc}}^{\hat{j}}}{\bar{P}_{j}}} .
$$

The result is plotted in Fig. 3 for two initial power noise values for the transfer and sensing beams. The plot shows that technical power noise and also shot noise of the transfer beam are suppressed even when considering a high $\mathrm{RPN}_{\mathrm{s}}=10^{-6} \mathrm{~Hz}^{-1 / 2}$, easily obtained with a traditional prestabilization, and a realistic amplifier gain of $|\epsilon|=10^{3}$. At low frequencies, the red curve is limited by technical radiation pressure noise from the sensing beam, while the blue curve is limited by the quantum radiation pressure noise. At high frequencies, both curves are limited by the interferometer readout noise. In the high loop gain regime, light will be squeezed when the absolute power noise of the sensing beam is smaller than 2 times the absolute shot noise of the transfer beam, i.e., $\mathrm{RPN}_{\mathrm{s}} \cdot \bar{P}_{\mathrm{s}}<2 \sqrt{2 \hbar \omega_{0} \bar{P}_{\mathrm{t}}}$.

Thermal noise is a particularly important noise source in this setup, especially for high susceptibility oscillators. The PSD of the micro-oscillator displacement caused by structural thermal noise is given by [13]

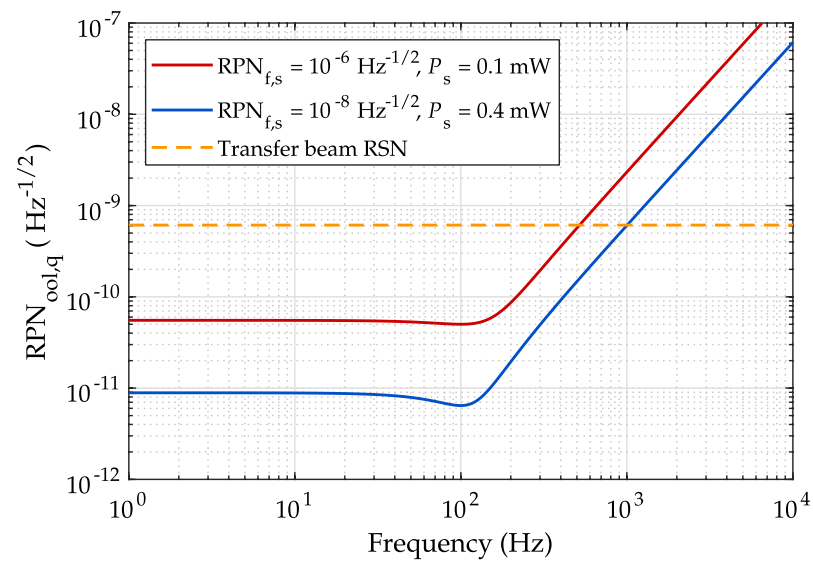

Fig. 3. ASD of $\mathrm{RPN}_{\text {ool, }}$ for different relative power noise in the transfer and sensing beams $\left(\mathrm{RPN}_{\mathrm{f}, \mathrm{s}}\right)$ and different sensing beam powers. For all curves, $\bar{P}_{\mathrm{t}}=1 \mathrm{~W}$ and $|\epsilon|=10^{3}$. The transfer beam relative shot noise (RSN) is depicted by the dashed yellow curve as reference. 


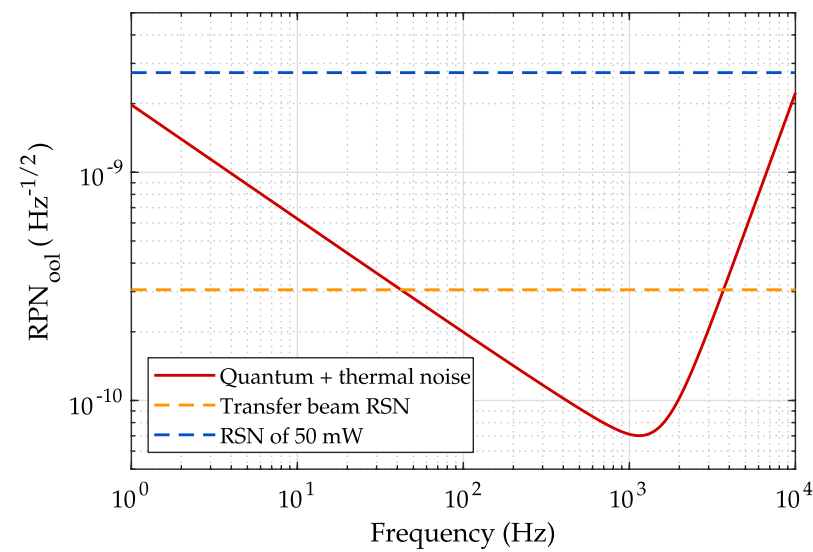

Fig. 4. ASD of the total $\mathrm{RPN}_{\text {ool }}$ (red curve) for $\bar{P}_{\mathrm{t}}=4 \mathrm{~W}$, $\bar{P}_{\mathrm{s}}=37 \mathrm{~mW}, \quad \mathrm{RPN}_{\mathrm{f}, \mathrm{s}}=10^{-8} \mathrm{~Hz}^{-1 / 2}, \quad T=4 \mathrm{~K}, \quad$ and $|\epsilon|=10^{3}$. The transfer beam relative shot noise is shown by the dashed yellow curve, and the relative shot noise of $50 \mathrm{~mW}$ (stability achieved with the traditional scheme) is shown by the dashed blue curve.

$$
x_{\mathrm{tn}}^{2}(\Omega)=\frac{4 k_{B} T \Omega_{0}^{2}}{m \Omega Q\left[\left(\Omega_{0}^{2}-\Omega^{2}\right)^{2}+\Omega_{0}^{4} / Q^{2}\right]},
$$

where $Q$ is the quality factor, $T$ is the temperature, and $k_{B}$ is the Boltzmann constant. The minimum relative power noise achievable in the out-of-loop beam when only limited by thermal noise $\left(\mathrm{RPN}_{\text {ool,tn }}\right)$ is equal to the power modulation that needs to be imprinted on the transfer beam to compensate the thermally driven motion of the micro-oscillator. This leads to

$$
\mathrm{RPN}_{\text {ool, tn }}=\frac{c \Omega_{0}}{\bar{P}_{\mathrm{t}}} \sqrt{\frac{k_{B} T m}{Q \Omega}} .
$$

This equation shows that the thermal noise contribution to $\mathrm{RPN}_{\text {ool }}$ scales with frequency in an opposite way than quantum noise, as it is larger for low frequencies and smaller for high frequencies. The parameter with most impact on improving the out-of-loop power stability in this case is the transfer beam power $\bar{P}_{\mathrm{t}}$. Decreasing the resonance frequency of the oscillator also improves the stability, but it restricts the stabilization frequency range to lower frequencies as well.

Figure 4 shows the total $\mathrm{RPN}_{\text {ool }}$ (red curve) obtained as an uncorrelated sum of the transfer scheme's quantum noise and thermal noise contributions. At frequencies below $1 \mathrm{kHz}$, the curve is limited by structural thermal noise, while for higher frequencies, it is limited by the interferometer readout shot noise. The value of $\bar{P}_{s}$ was chosen as an optimum compromise between readout shot noise and technical radiation pressure noise contributions. It is important to notice that for an alternative interferometer configuration in which the micro-oscillator is the end mirror for both interferometer arms [14], technical radiation pressure noise would not contribute to $\mathrm{RPN}_{\text {ool }}$. In this case, $\bar{P}_{\mathrm{s}}$ can be further increased, which reduces $\mathrm{RPN}_{\text {ool }}$ at high frequencies. Finally, the phase noise imprinted on the out-of-loop beam by the residual micro-oscillator motion is negligible in comparison with the free running phase noise of an nonplanar ring oscillator (NPRO) laser.
The micro-oscillator parameters chosen for this Letter were adjusted for an optimum stabilization aimed at frequencies higher than $10 \mathrm{~Hz}$. Similar oscillators have been reported in [15] (mass around $40 \mathrm{ng}$ ) and [16] (resonance frequencies around $150 \mathrm{~Hz}$ and quality factors of $Q=2 \times 10^{5}$ at $10 \mathrm{~K}$ ), meaning that an optimized oscillator might be available in the near future. An important and open question for these oscillators is the power damage threshold, which is a crucial parameter to the performance of the proposed scheme.

In summary, we calculated the fundamental limit for a nondemolition power stabilization scheme via radiation pressure. The results show a remarkable difference in comparison to the traditional and OAC-coupling schemes since a subshot noise power stabilization can be achieved. This is a consequence from the natural ponderomotive squeezing generated by the oscillator. We analyzed the scheme and its fundamental noises for realistic parameters and found that a bright squeezed beam with a power of $4 \mathrm{~W}$ and up to $12 \mathrm{~dB}$ of squeezing might be achievable in the near future. The corresponding $\mathrm{RPN}_{\mathrm{ool}}<6 \times 10^{-10} \mathrm{~Hz}^{-1 / 2}$ for frequencies between $10 \mathrm{~Hz}$ and $6 \mathrm{kHz}$ might be of interest for future gravitational wave detectors and other high-precision metrology experiments.

Funding. Deutsche Forschungsgemeinschaft (DFG, German Research Foundation) under Germany's Excellence Strategy-EXC-2123 Quantum Frontiers-390837967.

Acknowledgment. The authors thank $\mathrm{T}$. Corbitt for previous fruitful discussions.

Disclosures. The authors declare no conflicts of interest.

\section{REFERENCES}

1. G. M. Harry, LIGO Scientific Collaboration, Classical Quantum Gravity 27, 084006 (2010).

2. P. Kwee, B. Willke, and K. Danzmann, Opt. Lett. 34, 2912 (2009).

3. H. Vahlbruch, D. Wilken, M. Mehmet, and B. Willke, Phys. Rev. Lett. 121, 173601 (2018).

4. P. Kwee, B. Willke, and K. Danzmann, Opt. Lett. 33, 1509 (2008).

5. Y. Yamamoto, N. Imoto, and S. Machida, Phys. Rev. A 33, 3243 (1986).

6. K. Jacobs, P. Tombesi, M. J. Collett, and D. F. Walls, Phys. Rev. A 49, 1961 (1994).

7. S. L. Danilishin and F. Y. Khalili, Living Rev. Relativity 15, 5 (2012).

8. C. M. Caves and B. L. Schumaker, Phys. Rev. A 31, 3068 (1985).

9. B. L. Schumaker and C. M. Caves, Phys. Rev. A 31, 3093 (1985).

10. H. Kimble, Y. Levin, A. Matsko, K. Thorne, and S. Vyatchanin, Phys. Rev. D 65, 022002 (2001).

11. H. M. Wiseman and G. J. Milburn, Phys. Rev. A 49, 1350 (1994).

12. T. Corbitt, Y. Chen, F. Khalili, D. Ottaway, S. Vyatchanin, S. Whitcomb, and N. Mavalvala, Phys. Rev. A 73, 023801 (2006).

13. P. R. Saulson, Phys. Rev. D 42, 2437 (1990).

14. K. Yamamoto, D. Friedrich, T. Westphal, S. Goßler, K. Danzmann, K. Somiya, S. L. Danilishin, and R. Schnabel, Phys. Rev. A 81, 033849 (2010).

15. R. Singh, G. D. Cole, J. Cripe, and T. Corbitt, Phys. Rev. Lett. 117, 213604 (2016).

16. G. D. Cole, Proc. SPIE 8458, 845807 (2012). 